\title{
Sparse Ground Penetrating Radar Acquisition: Implication for Buried Landmine Localization and Reconstruction
}

\author{
Federico Lombardi ${ }^{\circledR}$, Member, IEEE, Hugh D. Griffiths ${ }^{\circledR}$, Fellow, IEEE, and Maurizio Lualdi ${ }^{\circledR}$
}

\begin{abstract}
The effectiveness of the ground penetrating radar (GPR) imaging process and its capability of correctly reconstructing buried objects is strictly bounded to a correct acquisition strategy, both in terms of data density and regularity. In some GPR applications, such as landmine detection, these requirements may not be fulfiled due to logistical limitations and environmental obstacles. In the light of autonomous platform, possibly driven by a positioning device, the knowledge of the maximum affordable grid irregularity is essential. This experimental work, employing a data set acquired at a landmine test site, provides a demonstration that the same information content could be maintained even with a sparser data grid, compared to the commonly adopted requirements, mitigating the pressing demand for a precise samples positioning.
\end{abstract}

Index Terms-Ground penetrating radar (GPR), landmine imaging, radar image reconstruction, trace positioning.

\section{INTRODUCTION}

$\mathbf{G}$ ROUND penetrating radar (GPR) is one of the most versatile sensing modalities currently employed for remote detection of buried objects [1]. Since it is based on the propagation and reflection of electromagnetic (EM) waves, it is sensitive to variations of the EM parameters in the subsoil, especially the dielectric constant and electric conductivity [2], [3]. By measuring the changes in dielectric constants, the usefulness of GPR is not limited by the metal content of the particular landmine [4]. GPR is beginning to be used as the main method to provide detection, but recognition and identification remains a challenge. This challenge could be mitigated if a properly sampled 3-D data set is available; however, the price to pay is a very high accuracy in trace positioning and acquisition regularity [5].

Although the significant advantages of 3-D surveying strategies are well documented, they generally require much greater acquisition expenditure and effort than that of traditional sparse bidimensional acquisitions. The 3-D imaging improvements with GPR have been tested in most domains of shallow

Manuscript received November 22, 2017; revised September 1, 2018; accepted September 14, 2018. This work was supported by the Find A Better Way Charity through the DETERMINE Program under Grant 2015/001D. (Corresponding author: Federico Lombardi.)

F. Lombardi and H. D. Griffiths are with the Department of Electronic and Electrical Engineering, University College London, London WC1E 6BT, U.K. (e-mail: f.lombardi@ucl.ac.uk).

M. Lualdi is with the Department of Civil and Environmental Engineering, Politecnico di Milano, 20133 Milan, Italy (e-mail: maurizio.lualdi@polimi.it).

Color versions of one or more of the figures in this letter are available online at http://ieeexplore.ieee.org.

Digital Object Identifier 10.1109/LGRS.2018.2872357 subsurface disciplines, from civil engineering to agriculture and archeology [6]-[8].

To obtain unaliased 3-D subsurface images, the spatial sampling must be sufficiently dense, at least a quarter wavelength both in the inline and crossline direction. Consequently, positioning precision needs to be better than $\lambda / 4$ for correct grid-point assignment. Anything worse than that may significantly decrease the accuracy of the scans [9]. This point is still hindering an extensive deployment of 3-D GPR in some critical environments.

As a logical outcome, 3-D manual GPR data acquisition is time consuming, labor intensive, and most of all, prone to positioning errors. The specific irregular positions can cause artifacts in the image and ultimately corrupt the final GPR image [10]. The choice of the positioning device for any survey depends upon the required accuracy, the extent of the survey area, the number of physical obstructions, and the level of background noise [11]-[13].

Given a Nyquist-rate spatial sampling and precise sample positioning, GPR data are commonly reconstructed by solving a linear interpolation problem, a method that ensures a low computational cost and proper performance. When the acquisition grid is excessively sparse, the interpolation process will inevitably introduce distortion [14].

Compressive sensing is one of the most recent techniques developed to reconstruct signals from fewer samples than is possible using the Nyquist sampling rate, by solving a nonlinear, convex optimization problem [15]. The central idea is to measure minimum samples that contain maximum information about the signal [16]. Although theoretically powerful, the technique has the drawback of being computational expensive.

In [17], a formulation of the sampling requirement which takes into account the depth of the target has been experimentally verified, showing that even for very shallow targets the Nyquist criterion is not an ironclad rule. In particular, the magnitude of the reduction factor in collected traces is not negligible: as a figure of merit, the ratio with the quarter wavelength criterion ranges from 1.3 to 2.4 samples. However, these results were obtained considering a regular grid acquisition, with a constant spacing between subsequent samples.

The aim of this letter is to advance the analysis by evaluating the limit of positioning errors to optimize 3-D GPR data acquisition to enhance the efficiency of GPR application for landmine detection. 


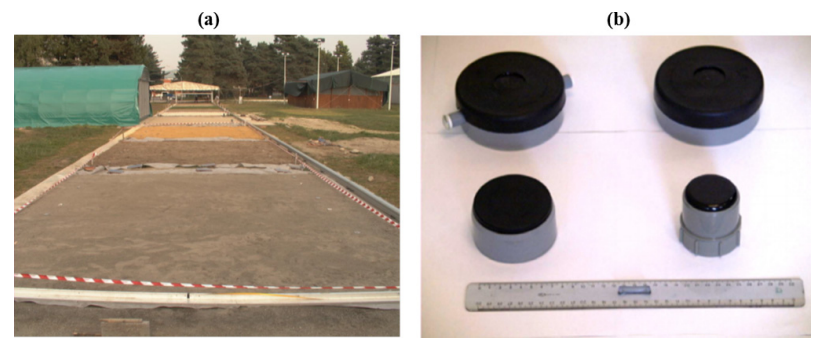

Fig. 1. Experimental test site. (a) View of the test lane. (b) Buried targets.

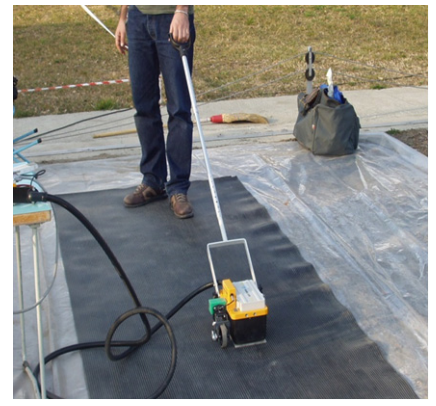

Fig. 2. Employed GPR equipment.

The structure of the remainder of this letter is as follows. Section II describes the experimental data set and the presented field trials, while the algorithm for generating the synthetic irregular grid is described and detailed in Section III. Section IV discusses the obtained results for each of the evaluated situations. Finally, conclusions are presented in Section V.

\section{EXPERIMENTAL CAMPAign Description}

The image presented in this letter have been acquired at the former multi-sensor mine-signature test site, located at the European Commission's Joint Research Centre, Ispra, Italy, consisting of a test lane including several adjacent bays filled with different soil materials. Each bay is populated with surrogate mines, with different size, metal content, and depth.

A general view of the lane and the buried targets is provided in Fig. 1.

For the experimentation, two different soil environments have been investigated: 1) a low loss loamy material, with a relative dielectric $\varepsilon$ of 4 and a resulting velocity of $15 \mathrm{~cm} / \mathrm{ns}$ and 2) a high losses clayed soil texture, characterized by a relative dielectric $\varepsilon$ of 10 and a propagation velocity of $9 \mathrm{~cm} / \mathrm{ns}$.

Target of interest was a surrogate of a large antipersonnel landmine with a diameter of $11 \mathrm{~cm}$ and buried at 5 and $15 \mathrm{~cm}$. The target [visible in the top right part of Fig. 1(b)] is designed to resemble mines in respect of their signatures and is molded in silicone rubber.

The GPR equipment employed, pictured in Fig. 2, was a shielded 1-GHz RAMAC/GPR equipment, manufactured by Mala Geoscience. The antenna consists of two bow-tie dipoles oriented perpendicular to the survey direction and separated $9 \mathrm{~cm}$.

Details on the acquired data and the acquisition parameters are provided in Table I.

Acquisition were carried out employing the pad system for georadar surveys [18] a solution which guarantees accurate
TABLE I

DATA Description AND ACQUisition PARAMETERS

\begin{tabular}{ll}
\hline \hline \multicolumn{1}{c}{ Parameter } & \multicolumn{1}{c}{ Value } \\
\hline Central frequency & $1 \mathrm{GHz}$ \\
Bandwidth & $1 \mathrm{GHz}$ \\
Time window & $10 \mathrm{~ns}$ \\
Inline sampling & $0.8 \mathrm{~cm}$ \\
Crossline sampling & $0.8 \mathrm{~cm}$ \\
Time sampling & $0.0913 \mathrm{~ns}$ \\
Acquired area $(\mathrm{x}, \mathrm{y})$ & $1 \times 1.30 \mathrm{~m}$ \\
Data samples $(\mathrm{x}, \mathrm{y}, \mathrm{t})$ & $120,160,110$
\end{tabular}

TABLE II

DATA PRocessing

\begin{tabular}{ll}
\hline \hline Parameter & Algorithm \\
\hline Time calibration & Time lag \\
Traces alignment & Static corrections \\
Frequency filtering & Zero phase Butterworth filter \\
Migration & Kirchhoff migration \\
Data display & Hilbert transform
\end{tabular}

data density and regularity, maintaining a precise profile spacing, and ensuring a constant antenna orientation during the whole survey [19]. The inline sampling accuracy was controlled by an odometric wheel directly connected to the sensor head.

Considering a maximum frequency of $1.5 \mathrm{GHz}$, the $\lambda / 4$ criterion for the loamy and clay material gives a maximum sampling interval of 2.5 and $1.5 \mathrm{~cm}$, respectively. As the purpose of the experimentation is to appraise the maximum affordable positioning error, the data were decimated following the outcomes of [17] to obtain the maximum acceptable sample interval and to create the worst possible scenario.

The processing applied to the data is described in Table II.

The migration algorithm, applied to focus the raw images and retrieve the spatial features of the data, is mathematically equivalent to the hyperbolic summation algorithm.

\section{IRREGULARITY GENERATION}

The synthetic positioning error, introduced on raw data (before the migration process) to effectively simulate a degraded acquisition, was computed by substituting the acquired sample (the nominal one) with an adjacent sample according to a definite but random criterion. The irregular grid has been created by randomly generating for each nominal sample a numeric flag indicating the axis and direction of the sample to replace the existing one. The chosen statistical distribution is a random uniform distribution, with a seed probability of 0.20 for each ill-positioned trace (4 in total) and 0.20 for the nominal sample [Fig. 3(a)].

The histogram of the distribution sequence is shown in Fig. 3(b).

The same error distribution was applied considering a different error radius [ $\delta$ in Fig. 3(a)], as defined in Table III.

The radius defines the distance with respect to the nominal position of the sample to be substituted, according to the geometry of Fig. 3(a). 


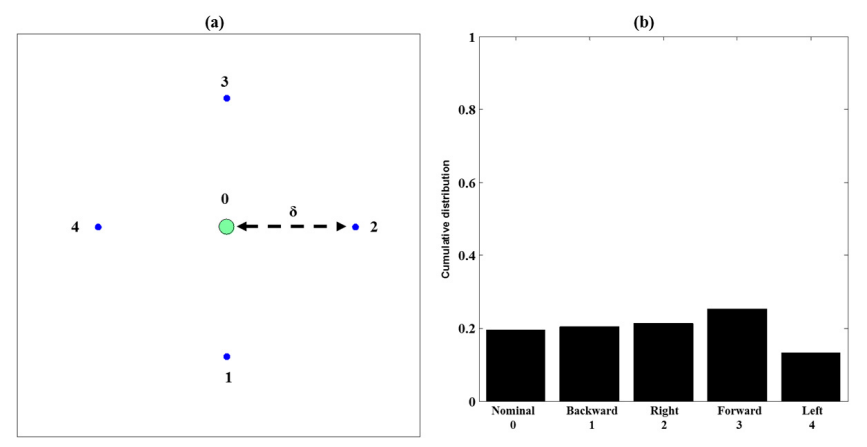

Fig. 3. Regularity degradation. (a) Sample replacement scheme. (b) Random distribution for traces substitution.

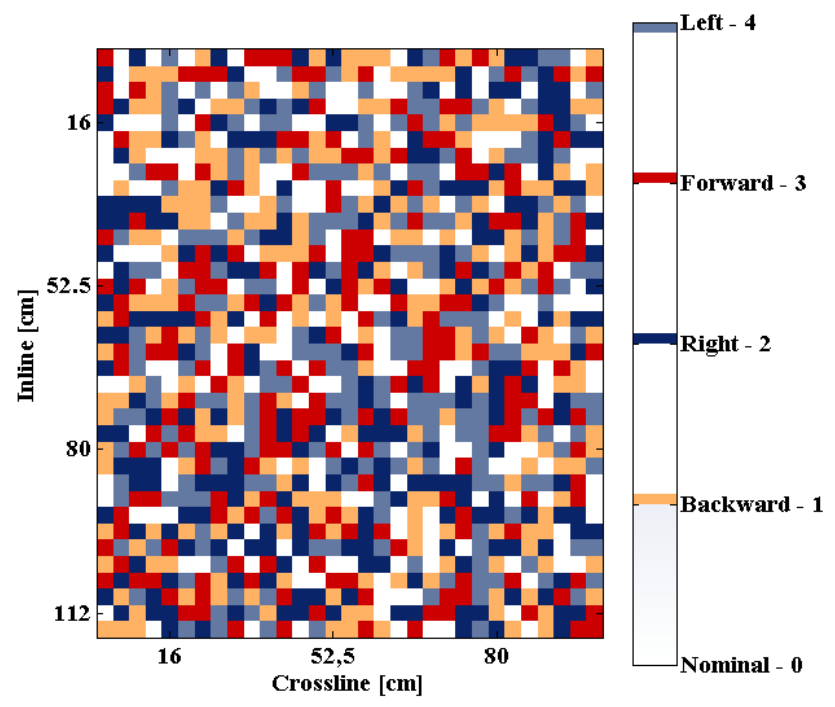

Fig. 4. Synthetic acquired grid after irregularity superimposition.

TABLE III

RADIUS OF MAXIMUM IRREGULARITY

\begin{tabular}{ccc}
\hline \hline Target depth & Radius, $\delta$ & $\begin{array}{c}\text { Distance from the } \\
\text { nominal sample }[\mathrm{cm}]\end{array}$ \\
\hline \multirow{3}{*}{$5 / 15 \mathrm{~cm}$} & 1 & 0.8 \\
& 2 & 1.6 \\
& 3 & 2.4 \\
& 4 & 3.2 \\
\hline
\end{tabular}

It can be seen from Fig. 3(a) that for each nominal sample, there is an equal probability of replacing it with the forward, backward, leftward, and rightward neighbor or maintain the correct one.

A sketch of the resulting irregular grid is shown in Fig. 4.

Such a distribution was chosen to simulate the most unpredictable situation in which: 1) the accuracy degradation is not polarized along a preferential direction and 2) no prediction of the possible spatial distribution of the acquisition error can be made in advance.

\section{TARget Reconstruction Results}

Data are displayed as a set of depth slices obtained by plotting the amplitude of the brightest reflector over the specified depth range at each scan location.

Moreover, to quantitatively assess the effect of the magnitude of the positioning error, the correlation between the (a)

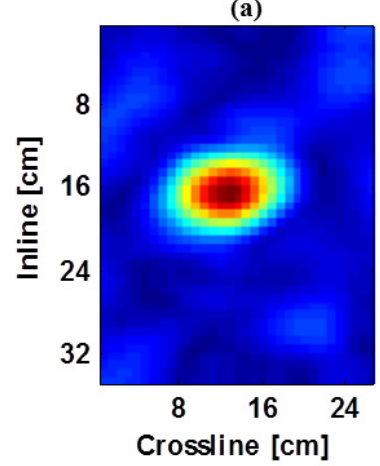

(c)

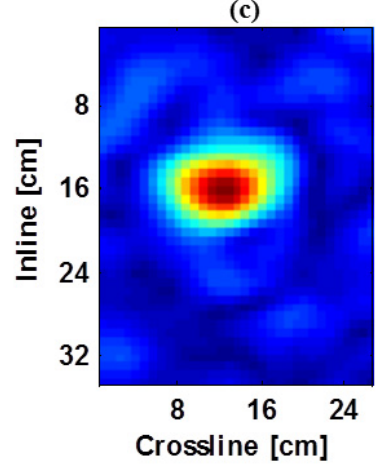

(e)

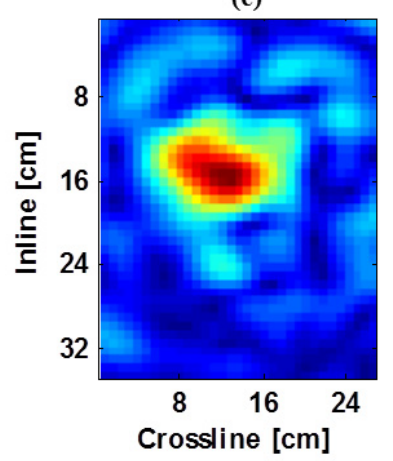

(b)

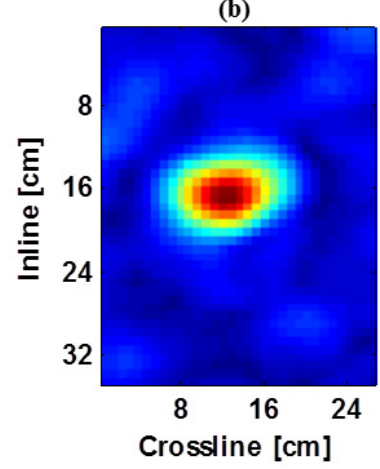

(d)

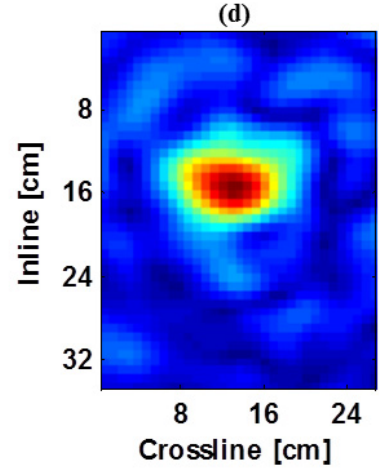

(f)

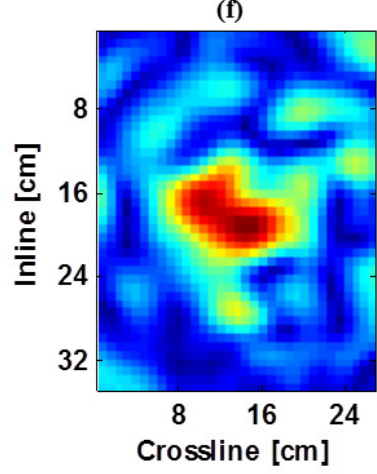

Fig. 5. Loamy soil, regularity degradation for the landmine buried at $5 \mathrm{~cm}$. Error radius (Table III). (a) 0 (regular). (b) 1. (c) 2. (d) 3. (e) 4. (f) 5.

regularly acquired slice and the irregular ones has been computed.

Of all the investigated targets, the set of depth slices are presented in full for the shallower targets, while the results from the landmines buried at $15 \mathrm{~cm}$ are shown through the correlation function.

\section{A. Loamy Soil Plot}

Processed depth slices for the landmine buried at $5 \mathrm{~cm}$ with varying error radius are presented in Fig. 5. Amplitude is displayed in a blue-yellow-red colour map and normalized in the range $[0-1]$ with respect to each relative maximum value. Irregularity radius is defined as per Table III.

Considering that the first frame, Fig. 3(a), is the original regular data, it can be seen that a pronounced degradation of the focusing performance arises from Fig. 3(c) onward, giving a maximum error radius of $1.6 \mathrm{~cm}$. This value corresponds to a half of the linear samples distance, suggesting that as long as 


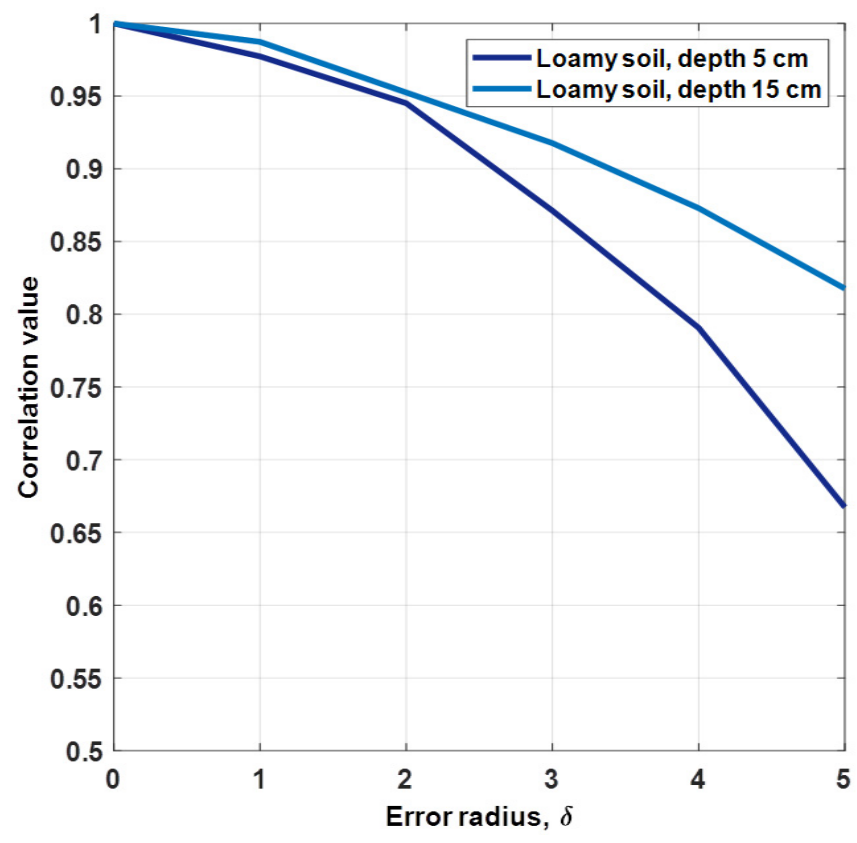

Fig. 6. Loamy soil plot, image correlation analysis.

the trace, even if misplaced, is included within the boundaries of the same information cell, defined as the Nyquist spacing for the given interval, no loss of information occurs and the reconstruction process is capable of correctly retrieving the spatial information.

Increasing the magnitude of inaccuracy causes processing artifacts faults which reduce the image quality. Obviously, the target is still detectable, thanks to the homogeneity of the host material, but the noise level could decrease the confidence in the recognition process.

The analysis of the images correlation between the regular and the degraded slices is provided in Fig. 6, together with the values obtained from the exploitation of the deeper target.

For the deeper target, given a larger affordable spacing, the ultimate limit of the irregularity corresponds to a radius of 2.4 $\mathrm{cm}$, consistent with the spacing of $4.8 \mathrm{~cm}$ of the regular data. This is identifiable as an increase of the inclination of curve after a radius of 3 . The same applies to the shallower target, for which the limit is definitely more evident. No significant losses of accuracy are evident elsewhere.

\section{B. Clay Soil Plot}

Fig. 7 presents the depth slices of the target buried at $5 \mathrm{~cm}$ in the clayed terrain.

Similar to what has been previously found; also in this case, a significant degradation of the image accuracy appears once the error radius equals half of the sampling interval. In particular, given a maximum sampling rate of $1.6 \mathrm{~cm}$ (both inline and crossline), degradation occurs starting from Fig. 7(c), in which processing noise becomes clearly visible.

However, differently from the loamy terrain, due to the higher absorption properties of the clay the target is better represented even for large irregularity magnitude. This is due to the reduction in the high-frequency content of the signal, (a)

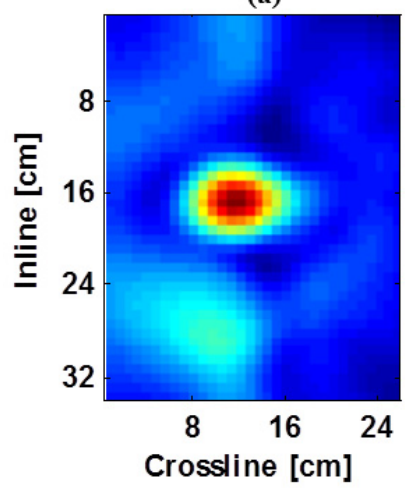

(c)

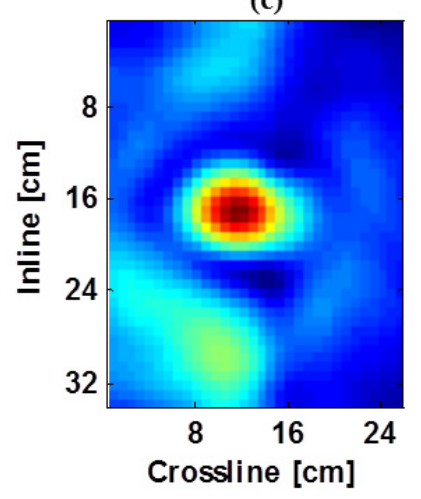

(e)
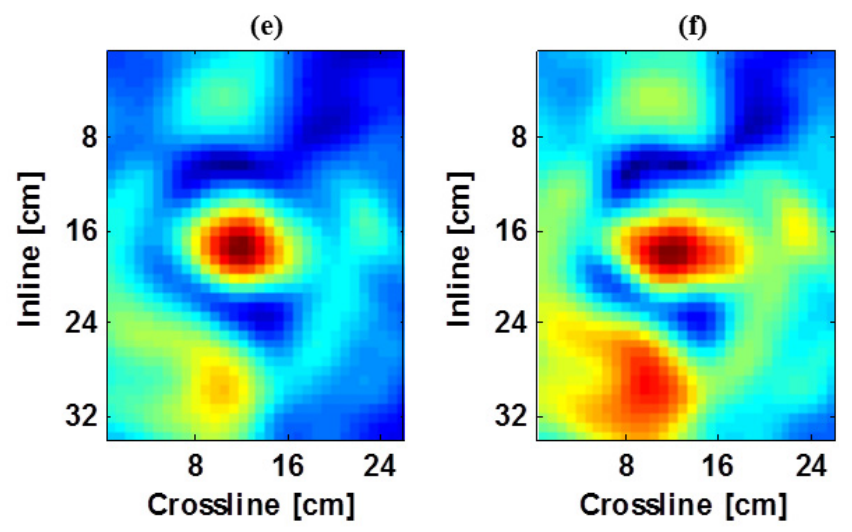

Fig. 7. Clay soil, regularity degradation for the landmine buried at $5 \mathrm{~cm}$. Error radius (Table III). (a) 0 (regular). (b) 1. (c) 2. (d) 3. (e) 4. (f) 5.

which is the portion that bounds the maximum sampling requirement. In conclusion, even if the material is theoretically more sensible to a regularity degradation, thanks to its lower velocity, the absorption contributes to a relaxation of this limit.

Fig. 8 shows the correlation analysis.

It can be noted from the graph that the correlation values for the clay soil environment are globally higher than in the previous scenario, and the curves are less sharp despite the expected behavior. The consequence is that in this case it is possible to define the boundary limit considering the correlation curve only for the shallower target, while for the landmine buried at $15 \mathrm{~cm}$ the pattern is almost flat.

The effect of the frequency absorption is even more evident comparing the behavior of the deeper target in the two environments. 


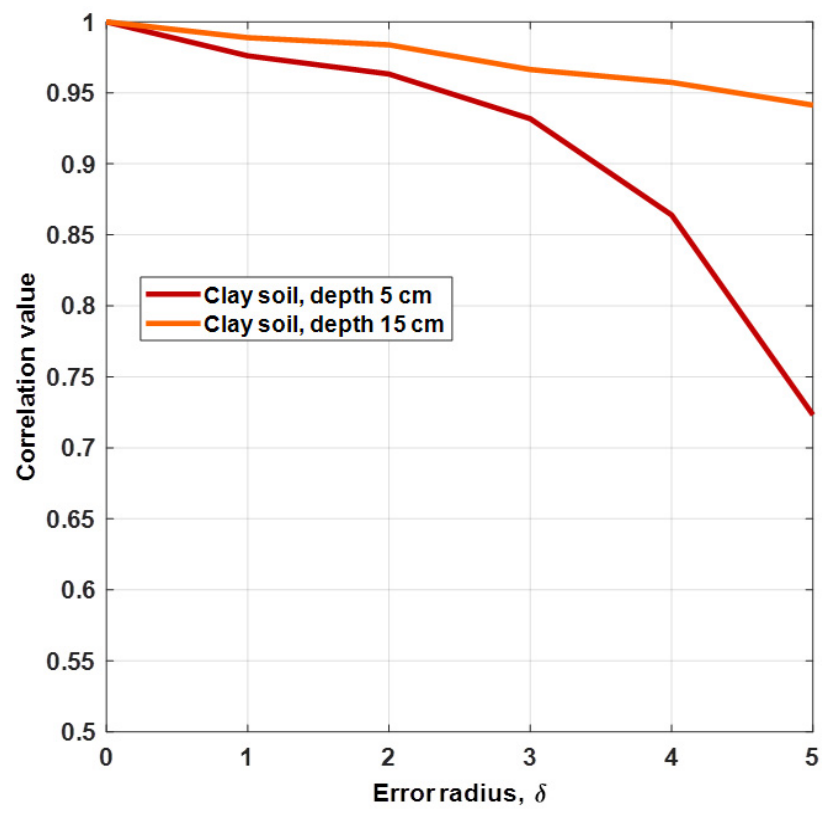

Fig. 8. Clay soil plot, image correlation analysis.

\section{CONCLUSION}

The need for autonomous devices with higher mobility is a continuous research topic for GPR applied to landmine detection. However, to obtain a clear and readable image, a 3D GPR acquisition should be carried out, meaning that precise and fine spatial samplings are needed. This logically affects the positioning devices, as it has to operate synchronously with the GPR and its accuracy should be definitely less than the GPR sample spacing.

The work starts from a set of previously obtained results on the topic of data density reduction, advancing the analysis by addressing the degradation of the positioning accuracy to explore the maximum radius of irregularity that the migration algorithm could not compensate for.

The acquisition grid was intentionally altered replacing the nominal sample with an adjacent one at a varying distance according to a specific random distribution. In this way, a synthetic irregular volume was obtained and processed. The scheme has been evaluated in different soil environments and at different landmine depth to jointly evaluate the two effects on the resulting GPR slices.

What has been found is that the boundary for a proper target reconstruction corresponds to half of the sampling distance, representing the dimension of the information cell included in the Nyquist rate. This means that as long as the linear samples are collected within this distance, there is no loss of information. Increasing the regularity degradation produces a radar image in which the target is still detectable, but its quality is reduced and the noise level could prevent the extraction of advanced features.

The experimental results taken together highlight that the same reconstruction performance that can be obtained with an uneven and coarser sampling grid, lowering the demanding, and challenging requirements of samples precision.

\section{REFERENCES}

[1] R. Bello, "Literature review on landmines and detection methods," Frontiers Sci., vol. 3, no. 1, pp. 27-42, 2013.

[2] N. F. E. Benedetto, M. Bano, A. Tzanis, J. Nyquist, K. Sandmeier, and N. Cassidy, "Advanced ground penetrating radar signal processing techniques," Signal Process., vol. 132, pp. 197-200, Mar. 2017, doi: 10.1016/j.sigpro.2016.07.032.

[3] M. A. Ghaderi, M. Dehmollaian, and A. Khaleghi, "Buried target imaging: A comparative study," Sens. Imag., vol. 18, p. 19, Dec. 2017, doi: 10.1007/s11220-017-0169-4.

[4] F. Lombardi, H. D. Griffiths, L. Wright, and A. Balleri, "Dependence of landmine radar signature on aspect angle," IET Radar, Sonar Navigat., vol. 11, no. 6, pp. 892-902, Jun. 2017.

[5] J. Groenenboom and E. C. Slob, "Sparse data acquisition and its influence on imaging," Proc. SPIE, vol. 4084, pp. 372-377, Apr. 2000.

[6] M. Lualdi and F. Lombardi, "Utilities detection through the sum of orthogonal polarization in 3D georadar surveys," Near Surface Geophys., vol. 13, no. 1, pp. 73-81, 2015.

[7] M. Molon, J. I. Boyce, and M. A. Arain, "Quantitative, nondestructive estimates of coarse root biomass in a temperate pine forest using 3-D ground-penetrating radar (GPR)," J. Geophys. Res., Biogeosciences, vol. 121, no. 12, pp. 80-102, 2016.

[8] W. Zhao et al., "Advances in GPR data acquisition and analysis for archaeology," Geophys. J. Int., vol. 202, no. 1, pp. 62-71, 2015.

[9] X. Feng, M. Sato, and C. Liu, "Hand-held GPR imaging using migration for irregular data," IEEE J. Sel. Topics Appl. Earth Observat. Remote Sens., vol. 4, no. 4, pp. 799-803, Dec. 2011.

[10] E. C. Slob, J. Groenenboom, and J. T. Fokkema, "Automated acquisition and processing of 3D GPR data for object detection and characterization," Subsurface Sens. Technol. Appl., vol. 4, no. 1, pp. 5-8, 2003.

[11] F. Lombardi, H. D. Griffiths, and A. Balleri, "Landmine internal structure detection from ground penetrating radar images," in Proc. IEEE Radar Conf., Oklahoma City, OK, USA, Apr. 2018, pp. 1201-1206.

[12] U. Boniger and J. Tronicke, "On the potential of kinematic GPR surveying using a self-tracking total station: Evaluating system crosstalk and latency," IEEE Trans. Geosci. Remote Sens., vol. 48, no. 10, pp. 3792-3798, Oct. 2010.

[13] N. Semeykin, V. Pomozov, and V. Monahov, "Integrated multi-channel unit for humanitarian mine-cleaning operations," in Proc. 15th Int. Conf. GPR, Brussels, Belgium, Jun./Jul. 2014, pp. 993-996.

[14] L. Yi, K. Takahashi, and M. Sato, "A fast iterative interpolation method in f-k domain for 3-D irregularly sampled GPR data," IEEE J. Sel. Topics Appl. Earth Observ. Remote Sens., vol. 9, no. 1, pp. 9-17, Jan. 2016.

[15] A. C. Gurbuz, O. Teke, and O. Arikan, "Sparse ground-penetrating radar imaging method for off-the-grid target problem," J. Electr. Imag., vol. 22, no. 2, p. $021007,2013$.

[16] E. Cristofani, M. Becquaert, S. Lambot, M. Vandewal, J. H. Stiens, and N. Deligiannis, "Random subsampling and data preconditioning for ground penetrating radars," IEEE Access, vol. 6, pp. 26866-26880, 2018.

[17] F. Lombardi, H. D. Griffiths, and M. Lualdi, "The influence of spatial sampling in GPR surveys for the detection of landmines and IEDs," in Proc. Eur. Radar Conf., London, U.K., Oct. 2016, pp. 322-325.

[18] M. Lualdi, “True' 3D acquisition using GPR over small areas: A cost effective solution," in Proc. Symp. Appl. Geophys. Eng. Environ. Problems, Charleston, SC, USA, 2011, pp. 541-550.

[19] F. Lombardi, H. D. Griffiths, and A. Balleri, "Bistatic radar signature of buried landmines," in Proc. Int. Conf. Radar Syst., Belfast, U.K., 2017, pp. 1-6. 